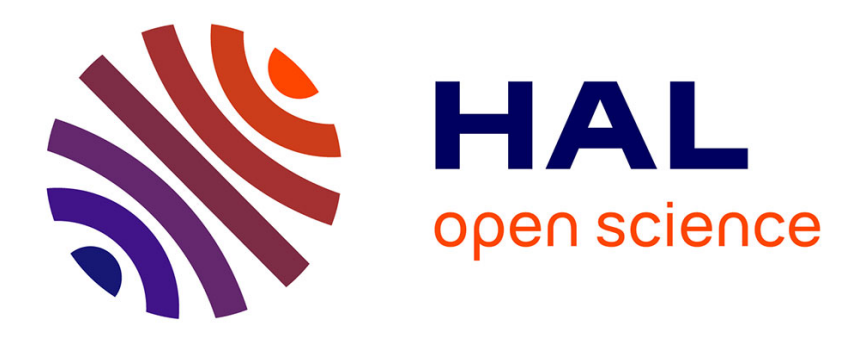

\title{
Angular particle sliding down a transversally vibrated smooth plane
}

Arnaud Benedetti, Philippe Sornay, Blanche Dalloz, Maxime Nicolas

\section{To cite this version:}

Arnaud Benedetti, Philippe Sornay, Blanche Dalloz, Maxime Nicolas. Angular particle sliding down a transversally vibrated smooth plane. Physical Review E: Statistical, Nonlinear, and Soft Matter Physics, 2012, 85 (1), pp.011307. 10.1103/PhysRevE.85.011307 . hal-01309378

\section{HAL Id: hal-01309378 https://hal.science/hal-01309378}

Submitted on 29 Apr 2016

HAL is a multi-disciplinary open access archive for the deposit and dissemination of scientific research documents, whether they are published or not. The documents may come from teaching and research institutions in France or abroad, or from public or private research centers.
L'archive ouverte pluridisciplinaire HAL, est destinée au dépôt et à la diffusion de documents scientifiques de niveau recherche, publiés ou non, émanant des établissements d'enseignement et de recherche français ou étrangers, des laboratoires publics ou privés. 


\title{
Angular particle sliding down a transversally vibrated smooth plane
}

\author{
Arnaud Benedetti \\ CEA, DEN, DEC, SPUA, LCU, F-13108 Saint-Paul-Lez-Durance, France and \\ Aix-Marseille Université, CNRS, IUSTI, UMR 6595, 5 rue Enrico Fermi, F-13453 Marseille cedex 13, France \\ Philippe Sornay \\ CEA, DEN, DEC, SPUA, LCU, F-13108 Saint-Paul-Lez-Durance, France \\ Blanche Dalloz and Maxime Nicolas \\ Aix-Marseille Université, CNRS, IUSTI, UMR 6595, 5 rue Enrico Fermi, F-13453 Marseille cedex 13, France
}

(Received 20 July 2011; published 25 January 2012)

\begin{abstract}
We present a theoretical, numerical, and experimental study about the sliding motion of an angular particle down a vibrated smooth plane. The model is based on a Coulomb's friction law with a unique friction coefficient. The model is solved numerically and is tested with controlled experiments. Different motion regimes are identified and the particle behavior is governed by two dimensionless parameters. The comparison between experimental and numerical results gives an indirect access to the dynamic friction coefficient.
\end{abstract}

DOI: 10.1103/PhysRevE.85.011307

PACS number(s): 45.70.-n, 46.55.+d

\section{INTRODUCTION}

The handling of powders or granular matter is a major issue for many industries. A large amount of granular matter is handled worldwide by the building industry (cement, sand, gravel), the pharmaceutical industry (excipient powders), agrochemical (seeds, nutriments), food industry (wheat, starch, cereals), moulding processes (metallic powders, plastics, ceramics). Among the numerous physical and mechanical parameters that describe the powder, the flowability is a complex parameter arising from the interactions between particles, and from the interactions of the particles with the substrate. The flowability is obviously affected by the nature of contacts and forces between adjacent particles. In addition of weak adhesive forces (especially van der Waals or electrostatic forces), the geometry and roughness of particle surfaces influence strongly the flowability. This problem is acute for nonconvex particles where particle imbrication may lead to clusters formation and blockage. While a spherical model for the particle shape is convenient for many calculations and computations, it is far from realistic for many actual powders.

When the handling is driven by gravity, the friction coefficient between the particles and the walls or the substrate is a governing parameter. Despite the great difficulty to understand the friction coefficient at a fundamental level [1], it is a unique parameter in the simple Coulomb's law. Due to the strong nonlinearity of the Coulomb's friction law, the response of a sliding block submitted to various external solicitations has been the subject of numerous studies. The motion of a block on a horizontal vibrated substrate has long attracted earthquake and building engineers [2,3]. Since the works of Newmark [4], the sliding block model is widely used to understand the dynamics of landslides along inclined soil failures subjected to a seismic periodic loading [5]. The coupling between friction and vibration may lead to friction-induced oscillations, leading to squeaking noises [6].

Since the friction hinders the motion and displacement of granular particles, external mechanical vibrations have been proven as a good technical solution to increase the flowability. On a horizontal plane, asymmetric vibrations may lead to a net transport velocity; this behavior has been studied in detail by Buguin et al. [7] and Fleishman et al. [8] for a single block. Considering symmetric vibrations, Sanchez et al. [9] studied the spreading of a granular pile on a vibrated horizontal plate.

More recently, Méndez et al. [10] have studied the trajectory of a sliding block down a plane with a longitudinal vibration. A parametric dynamic friction coefficient has been introduced to model the behavior of the block, putting aside the simplicity of a constant friction coefficient in a mechanical model.

In this paper we present in Sec. II a simple model of an angular solid grain sliding down an inclined and vibrated smooth plane. Due to its angular shape and its aspect ratio, the particle does not rotate while sliding down the plane. The inclination is characterized by an angle $\theta$, while the friction force between the grain and the plane is modeled with a simple Coulomb's law with a unique friction coefficient $\mu$. The vibration axis is perpendicular to the longitudinal axis of the plane, projection of the gravity field vector.

From the movement equation we can identify three regimes: a stick regime where the grain has no relative motion to the plane, a stick-slip regime when a sliding motion is periodically observed, and a pure-slip regime where the grain never sticks. The movement equation is solved numerically in Sec. III and the main features of the grain trajectory, such as the transverse amplitude and the mean longitudinal velocity, are measured. After a presentation of the experimental setup (Sec. IV), the experimental data are compared with the numerical results in Sec. V and a conclusion closes the paper.

\section{THEORETICAL MODEL}

We consider a block lying on an inclined plane with an angle $\theta$ to the horizontal (see Fig. 1). The plane is transversally 


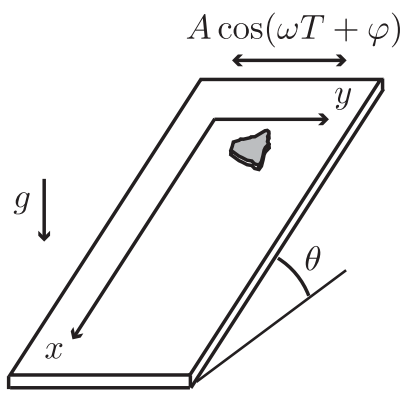

FIG. 1. Sketch of the setup.

vibrated in the $y$ direction with a single frequency $\omega / 2 \pi$ and an amplitude $A$. In the plane reference frame, the velocity $\mathbf{V}$ is governed by

$$
m \frac{d \mathbf{V}}{d T}=m g \sin \theta \mathbf{e}_{x}+\mathbf{F}-m A \omega^{2} \cos (\omega T+\varphi) \mathbf{e}_{y},
$$

where $\mathbf{e}_{x}$ and $\mathbf{e}_{y}$ represent the base vectors of the plane, and $m$ the particle mass. The friction force $\mathbf{F}$ between the block and the plane is modeled by a simple Coulomb's law with a unique friction coefficient $\mu=\tan \theta_{c}$ where $\theta_{c}$ is the angle of friction: $\mathbf{F}=-\mu m g \cos \theta \mathbf{V} /\|\mathbf{V}\|$ when the velocity is nonzero. In expression (1) $\varphi$ is an arbitrary phase.

Using the dimensionless time and velocity variables

$$
t=T\left(\frac{g \cos \theta}{A}\right)^{1 / 2}, \quad \mathbf{v}=\frac{\mathbf{V}}{\sqrt{A g \cos \theta}},
$$

the governing equation (1) writes

$$
\frac{1}{\mu} \frac{d \mathbf{v}}{d t}=\beta \mathbf{e}_{x}-\frac{\mathbf{v}}{\|\mathbf{v}\|}-\alpha \cos (\sqrt{\alpha \mu} t+\varphi) \mathbf{e}_{y},
$$

with an angle parameter $\beta$ and a vibration parameter $\alpha$ defined as

$$
\beta=\frac{\tan \theta}{\mu}, \quad \alpha=\frac{A \omega^{2}}{\mu g \cos \theta} .
$$

In dimensionless form, the sticking condition is written as

$$
\left(\frac{1}{\mu} \frac{d v_{x}}{d t}+\beta\right)^{2}+\left(\frac{1}{\mu} \frac{d v_{y}}{d t}-\alpha \cos (\sqrt{\alpha \mu} t+\varphi)\right)^{2}<1,
$$

meaning that the block is stuck on the plane when the vector sum of inertia and gravity forces is lower than the friction force.

\section{A. Stick, slip, and stick-slip regimes}

When the sticking condition (5) is valid at any time, both velocity and acceleration are zero, thus the sticking condition is $\beta^{2}+\alpha^{2} \cos ^{2}(\sqrt{\alpha \mu} t)<1$, which is true at any time for any $\alpha$ below a critical value

$$
\alpha_{\text {stick }}=\sqrt{1-\beta^{2}} .
$$

Following Westermo [3], a permanent sliding regime can be found. According to Eq. (5), the beginning of the sliding motion occurs at a time $t_{0}$, such as

$$
\sqrt{\alpha \mu} t_{0}=\arccos \left(\frac{\sqrt{1-\beta^{2}}}{\alpha}\right) \text {. }
$$

Assuming that the acceleration vanishes at the beginning and at the end of the sliding motion (at $t_{0}$ and $t_{f}$ ), we have from the $x$ component of Eq. (3)

$$
\beta=\left(\frac{v_{x}}{\|\mathbf{v}\|}\right)_{t=t_{0}, t_{f}}
$$

hence, since $\|\mathbf{v}\|=\sqrt{v_{x}^{2}+v_{y}^{2}}$,

$$
\frac{v_{y}}{\|\mathbf{v}\|}=\sqrt{1-\beta^{2}} \text {. }
$$

Integrating the $y$ component of Eq. (3) and using Eq. (9) gives

$$
\left(t_{f}-t_{0}\right)\left(1-\beta^{2}\right)^{1 / 2}=\sqrt{\frac{\alpha}{\mu}}\left[\sin \left(\sqrt{\alpha \mu} t_{0}\right)-\sin \left(\sqrt{\alpha \mu} t_{f}\right)\right] .
$$

The sliding motion never stops when the sliding duration $t_{f}-$ $t_{0}$ equals a half vibration period $\tau / 2=\pi / \sqrt{\alpha \mu}$. In that case $\sin \left(\sqrt{\alpha \mu} t_{f}\right)=-\sin \left(\sqrt{\alpha \mu} t_{0}\right)$ and finally we obtain

$$
\arccos \left(\frac{\sqrt{1-\beta^{2}}}{\alpha}\right)=\arcsin \left(\frac{\pi}{2} \frac{\sqrt{1-\beta^{2}}}{\alpha}\right)
$$

and the permanent sliding motion (permanent slip regime) occurs for any $\alpha$ larger than

$$
\alpha_{\text {slip }}=\alpha_{\text {stick }} \sqrt{1+\pi^{2} / 4} \approx 1.862 \alpha_{\text {stick }} .
$$

Expressions (6) and (10) are drawn in a $(\beta, \alpha)$ plane in Fig. 2. These two curves separate the space in three regions: the permanent stick regime where the block has no relative motion to the substrate, the stick-slip regime where the block has an intermittent sliding motion, and the permanent slip regime where the block is continuously sliding.

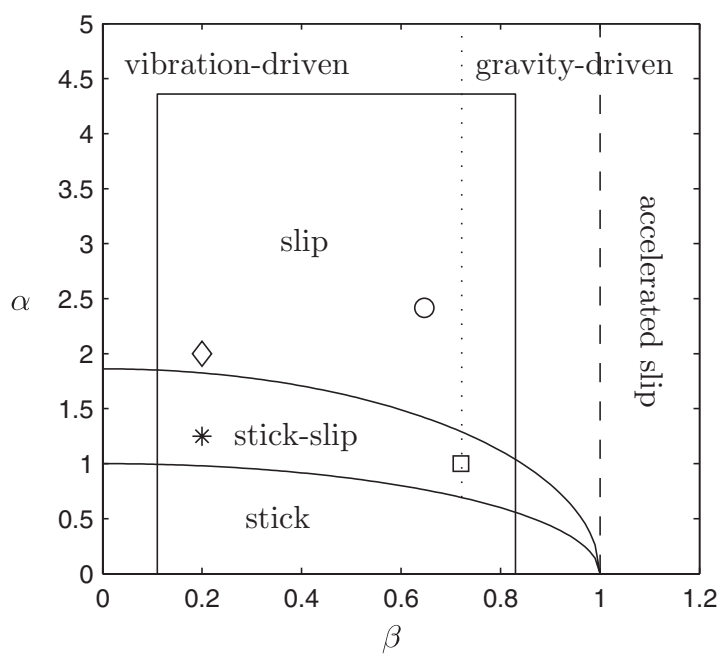

FIG. 2. Map of the different regimes in the $(\beta, \alpha)$ plane. For inclination angle above the friction angle $(\beta>1)$, an accelerated slip regime (a.s.r.) is expected. The rectangle indicates the range accessible experimentally for a static friction angle of $40^{\circ}$. The dotted line separates the vibration-driven regime and the gravity-driven regime. The star refers to the trajectory presented in Fig. 3 and the diamond refers to the trajectory presented in Fig. 4. The empty circle and the square symbol refer to experimental and numerical results presented in Figs. 11 and 12 in Sec. V (permanent slip and stick-slip regimes, respectively). 


\section{B. Energy balance for periodic solutions}

During the motion, Eq. (3) holds without condition. Multiplying by $\mathbf{v}$ gives

$$
\frac{1}{2 \mu} \frac{d v^{2}}{d t}-\beta v_{x}=-\|\mathbf{v}\|-\alpha \cos (\sqrt{\alpha \mu} t+\varphi) v_{y}
$$

or

$$
\begin{aligned}
& \frac{1}{2 \mu \tau}\left[v^{2}(t+\tau)-v^{2}(t)\right]-\frac{1}{\tau} \int_{t}^{t+\tau} \beta v_{x} d t \\
& +\frac{1}{\tau} \int_{t}^{t+\tau}\|\mathbf{v}\| d t+\frac{1}{\tau} \int_{t}^{t+\tau} \alpha \cos (\sqrt{\alpha \mu} t+\varphi) v_{y} d t=0
\end{aligned}
$$

Assuming that the velocity $\mathbf{v}$ is a periodic function after the transient motion from rest, the variation of $v^{2}$ between $t$ and $t+\tau$ is thus zero and Eq. (11) reduces to

$$
\frac{1}{\mu}\left(\bar{E}_{p}+\bar{E}_{d}+\bar{E}_{i}\right)=0
$$

with the time-averaged potential and the dissipated and injected energies defined as

$$
\begin{gathered}
\bar{E}_{p}=\beta \mu \int_{t}^{t+\tau} v_{x} d t=\beta \mu \tau \bar{v}_{x}, \\
\bar{E}_{d}=-\mu \int_{t}^{t+\tau}\|\mathbf{v}\| d t, \\
\bar{E}_{i}=-\alpha \mu \int_{t}^{t+\tau} \cos (\sqrt{\alpha \mu} t+\varphi) v_{y} d t .
\end{gathered}
$$

These averaged energies are numerically computed and the results are presented as powers $\bar{E}_{p} / \tau, \bar{E}_{d} / \tau$, and $\bar{E}_{i} / \tau$ in the next section.

\section{NUMERICAL METHOD AND RESULTS}

\section{A. Numerical method}

Equations (3) and (5) are implemented in a MATLAB code using an implicit scheme. The numerical method has been tested with various classical configurations without the transverse vibration. In the stick-slip mode a time step of $10^{-4}$ was chosen to capture accurately the beginning and end of each sliding phase. In the slip mode, a time step of $10^{-3}$ was sufficient.

\section{B. Typical trajectories}

A typical block trajectory is shown in Fig. 3 illustrating the stick-slip mode for $\alpha=1.25$ and $\beta=0.2$. The trajectory is shown in the plane reference frame (a) and in the laboratory reference frame (b). The grain is stuck on the plane until the sum of the weight and the inertia force overcomes the friction force. The onset of motion is indicated by an empty circle on the trajectory. When the grain slides, the trajectory is mainly downward oriented, and its kinetic energy is dissipated by the friction with the substrate. The relative motion stops when the friction force overcomes the other forces, and this is indicated by a solid black disk. After a short transient $(0<t<3)$, the trajectory reaches a periodic regime with an alternation of stick and slip phases.
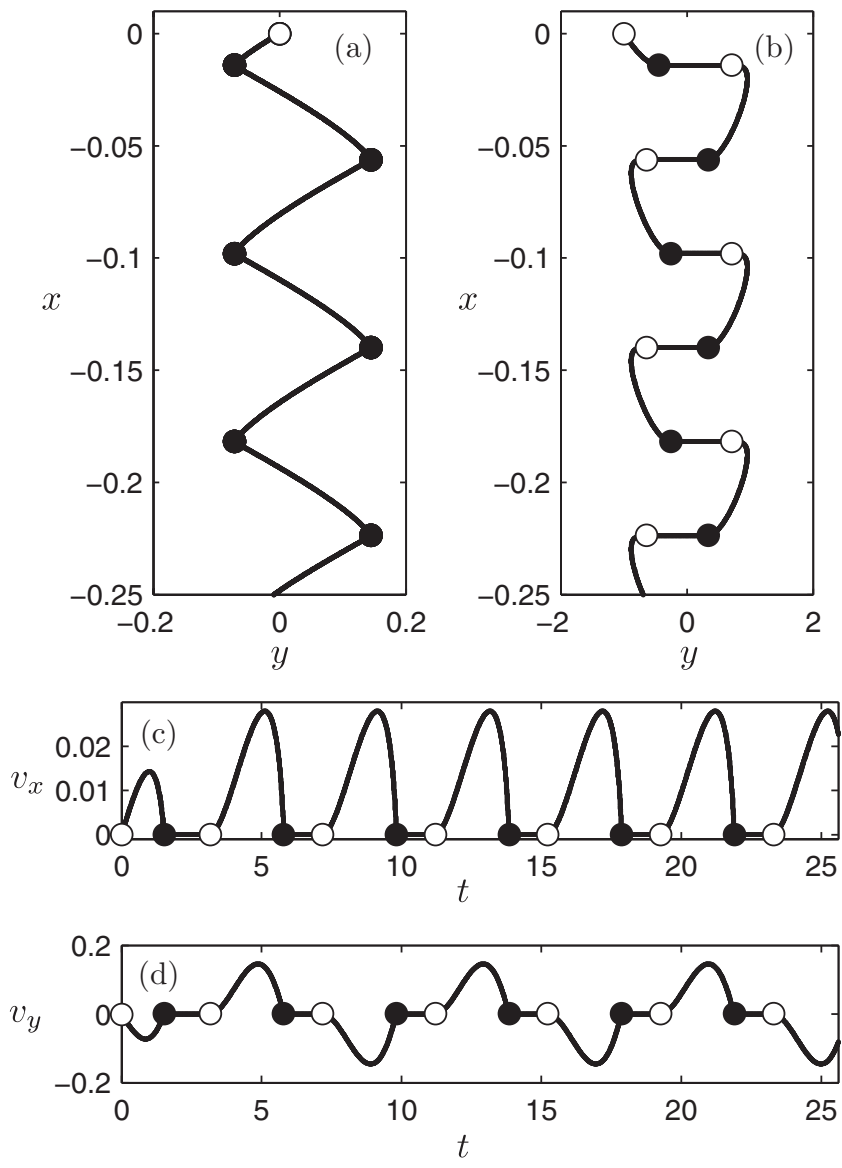

FIG. 3. Example of a trajectory of the block in the stick-slip regime in the plane reference frame (a) and in the laboratory reference frame (b). The corresponding velocity evolution $\left(v_{x}, v_{y}\right)$ is shown in (c) and (d). The empty circles indicate the beginning of the sliding phase, and conversely the solid black disks indicate the end of the sliding phase. Vibration and angle parameters are $\alpha=1.25$ and $\beta=0.2$ (see the star symbol in Fig. 2).

Also shown in Fig. 3 are the velocity components $v_{x}$ (c) and $v_{y}(\mathrm{~d})$ of the particle. Obviously the downward velocity $v_{x}$ is either zero or positive whereas the transverse velocity $v_{y}$ has a zero mean value after the transient has vanished.

Figure 4 illustrates the permanent slip mode, where the sticking condition (5) is never valid except during a short transient at the beginning of the motion. After the transient, the trajectory reaches a periodic regime, as in the stick-slip regime. In this regime the friction force is never strong enough to overcome other forces and the block slides downward continuously with a nonzero velocity.

\section{Block vibration amplitude and its mean downward velocity}

Several quantities can be computed from the numerical trajectory. Since the trajectory is periodic, it is easy to compute the block vibration amplitude such as $|y| \leqslant A_{b}$ and the mean downward velocity $\bar{v}_{x}$ as defined in Eq. (13).

The block vibration amplitude $A_{b}$ (computed in the laboratory reference frame) is shown in Fig. 5(a) with a continuously varying angle parameter and five typical values of the vibration parameter. The limit $A_{b}=1$ means that the grain is stuck on 

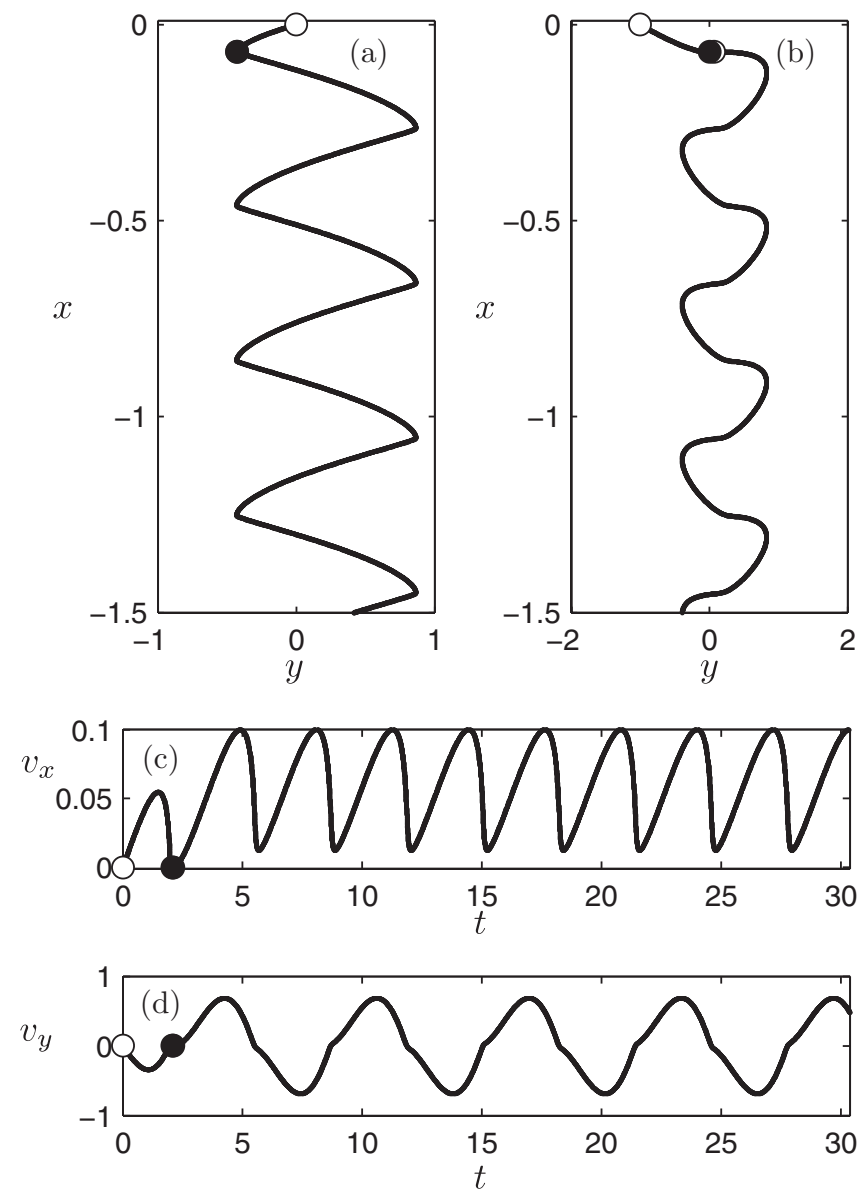

FIG. 4. Example of a trajectory of the block in the permanent slip regime in the plane reference frame (a) and in the laboratory reference frame (b). The corresponding velocity evolution $\left(v_{x}, v_{y}\right)$ is shown in (c) and (d). The empty circles indicate the beginning of the sliding phase, and conversely the solid black disks indicate the end of the sliding phase. After a transient $(t>3)$, no stop occurs. Vibration and angle parameters are $\alpha=2$ and $\beta=0.2$ (see the diamond symbol in Fig. 2).

the plane in the stick regime. When $\beta$ increases, the block amplitude decreases, and eventually goes to zero for $\beta>1$, where the block experiences a permanently accelerated regime where the weight dominates the vibration force. The amplitude $A_{b}$ also depends on the vibration parameter $\alpha$ as shown on Figs. 5(a) and 6(a). An increase in the vibration intensity decreases the grain amplitude since the vibration helps the block to slide down the substrate.

The block mean downward velocity $\bar{v}_{x}$ [Fig. 5(b)] varies with the angle parameter and increases strongly when $0.8<$ $\beta<1$. This velocity diverges obviously when $\beta \rightarrow 1$ when the block approaches the accelerated regime. Plotting $\bar{v}_{x}$ versus $\alpha / \alpha_{\text {stick }}-1$ shows that the mean velocity is close to zero for $\left(\alpha / \alpha_{\text {stick }}-1\right) \lesssim 0.5$, then increases nonlinearly [Fig. 6(b)].

\section{Energies}

In the periodic regimes, the time-averaged energies are computed for any $(\alpha, \beta)$ parameters from expressions (13)-(15). Figure 7 shows the variation of these energies when $\alpha$ increases from 0 . For a low plane inclination $(\beta=0.1)$,
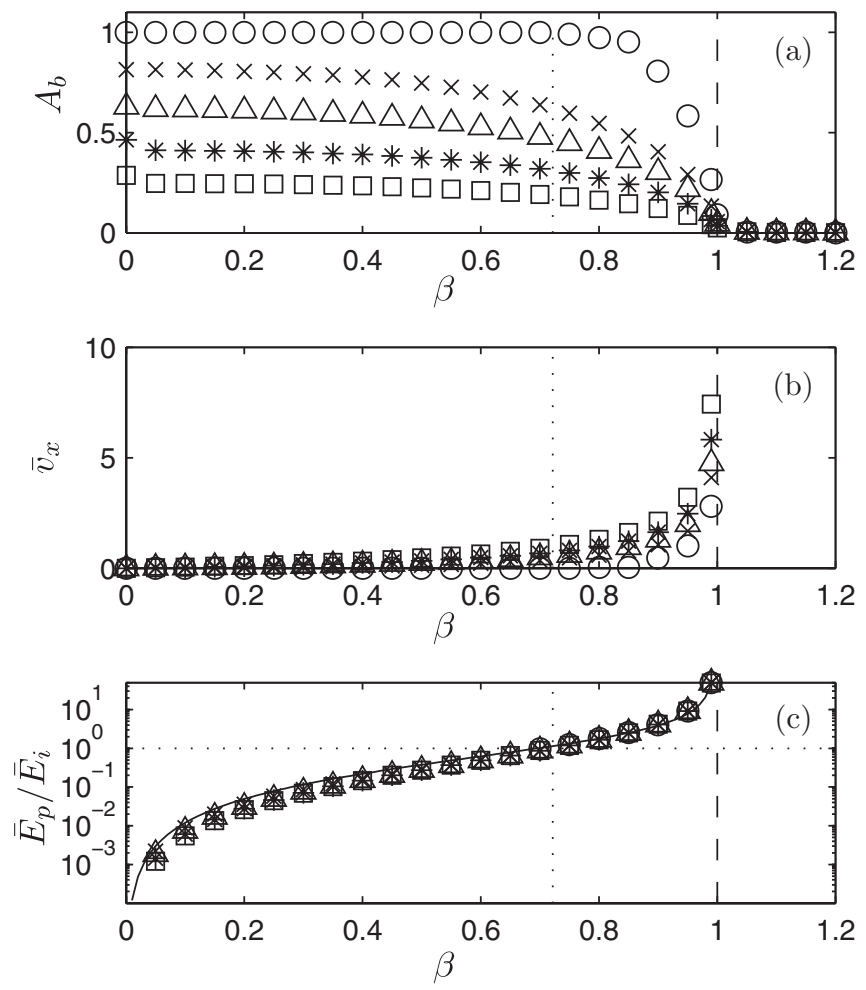

FIG. 5. (a) Vibration amplitude of the block varying with $\beta$ for different values of the vibration parameter: $\alpha=0.75$ (circles), 1.5 (crosses), 2 (triangles), 3 (stars), 5 (squares). (b) Plot of the mean downward velocity $\bar{v}_{x}$. (c) Ratio of the potential to injected averaged energies. The solid line is the empirical expression $H(\beta)=$ $3.6\left\{\exp \left[\left(1-\beta^{2}\right)^{-1 / 3}-1\right]-1\right\}$. For the three plots the dotted line separates the vibration-driven regime (left) from the gravity-driven regime (right).

the potential energy is negligible compared to injected energy, which is balanced by friction dissipated energy. As the inclination angle increases (for example, for $\beta=0.5$ ) the potential energy is no longer negligible. For a large inclination angle the potential energy overcomes the injected energy.

Figure 8 shows the variation of the averaged energies with the angle parameter $\beta$. One can notice that the $\bar{E}_{p}$ and $\bar{E}_{d}$ curves cross for a critical value $\beta^{*} \approx 0.722$. This critical value appears to be independent from the vibration parameter $\alpha$. Therefore we can define a vibration-driven regime for $\beta<\beta^{*}$ and a gravity-driven regime for $\beta>\beta^{*}$. The critical value $\beta^{*}$ is shown as a dotted line in Fig. 5.

As can be seen in Fig. 5(c), the energy ratio $\bar{E}_{p} / \bar{E}_{i}$ is a function of the angle parameter $\beta$ only and does not depend on the vibration parameter $\alpha$ despite $\bar{E}_{p}$ and $\bar{E}_{i}$ being functions of $\alpha$ :

$$
\frac{\bar{E}_{p}}{\bar{E}_{i}}=-\frac{\beta}{\alpha} \frac{\int_{t}^{t+\tau} v_{x} d t}{\int_{t}^{t+\tau} \cos (\sqrt{\alpha \mu} t+\varphi) v_{y} d t}=H(\beta) .
$$

This ratio tends to zero for a small inclination angle, and diverges when the inclination angle reaches the friction angle.

\section{EXPERIMENTAL SETUP}

The experimental setup is drawn as a sketch in Fig. 1. The inclined plane is an mirror-polished stainless-steel board of 

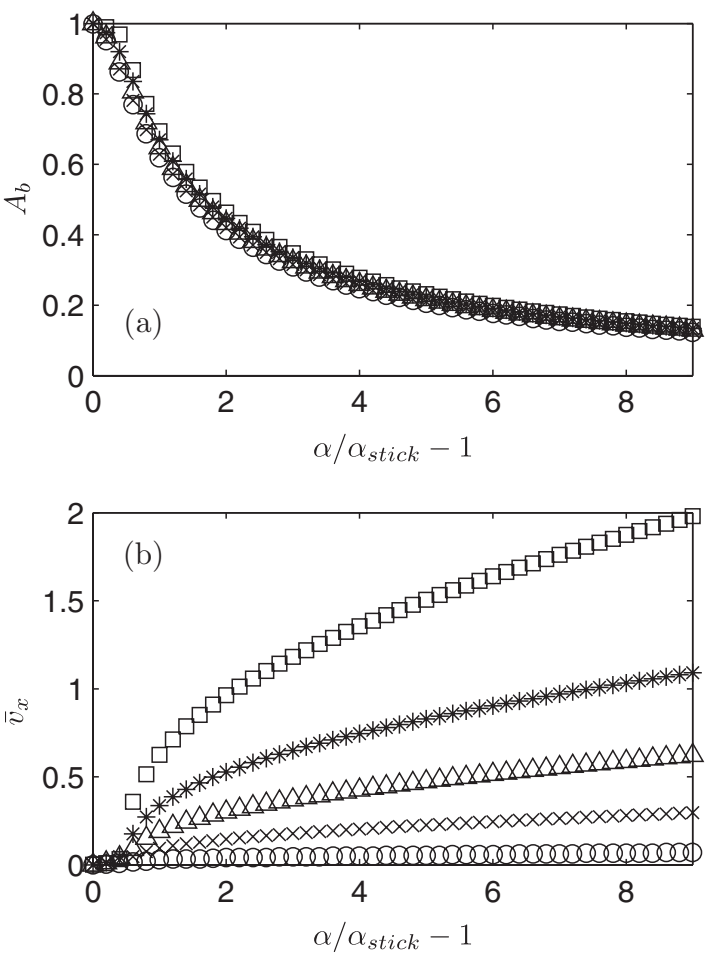

FIG. 6. Block amplitude dependence on the vibration parameter $\alpha$ (a) and mean downward velocity depending on the vibration parameter $\alpha$ (b). For plots (a) and (b) the symbols are $\beta=0.1$ (circles), 0.3 (crosses), 0.5 (triangles), 0.7 (stars), and 0.9 (squares).

size $40 \times 20 \mathrm{~cm}^{2}$. The plane inclination can be varied from 0 to $35^{\circ}$ with an accuracy of $\pm 0.2^{\circ}$. The plane is mounted on a horizontal sliding rail and rigidly connected to a permanent magnet shaker (LDS V406-PA driven by a 500L amplifier). We checked that the plane vibration was one dimensional and monochromatic. The maximum acceleration was varied from
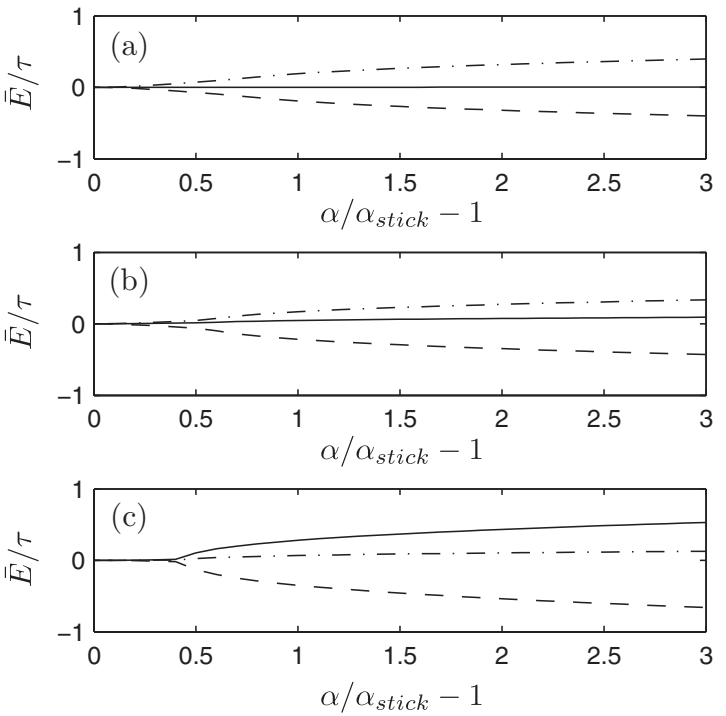

FIG. 7. Time-averaged energies per time unit (powers). (a) $\beta=$ 0.1 , (b) $\beta=0.5$, (c) $\beta=0.9$. Potential energy $\bar{E}_{p}$ : continuous line; injected energy $\bar{E}_{i}$ : dash-dot line; dissipated energy $\bar{E}_{d}$ : dashed line.
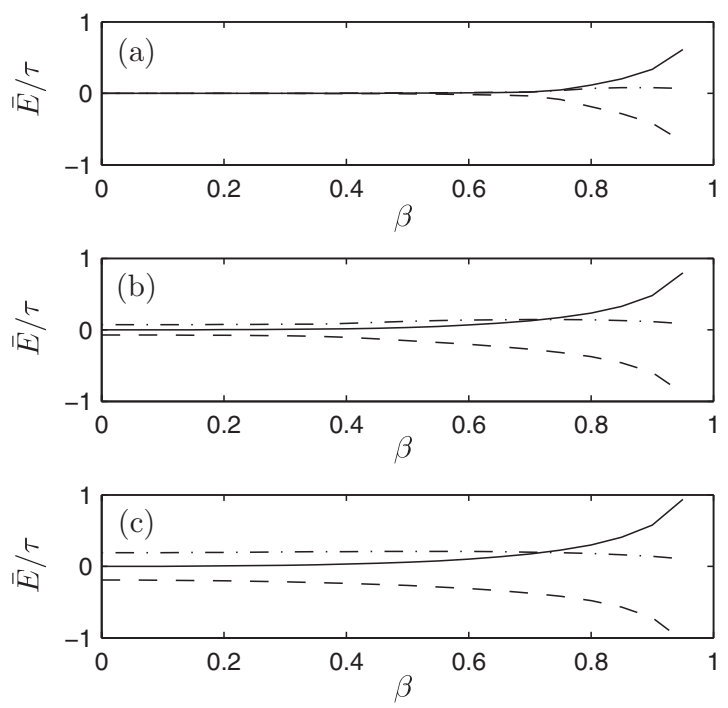

FIG. 8. Time-averaged energies per time unit (powers). (a) $\alpha=1$, (b) $\alpha=1.5$, (c) $\alpha=2$. Potential energy $\bar{E}_{p}$ : continuous line; injected energy $\bar{E}_{i}$ : dash-dot line; dissipated energy $\bar{E}_{d}$ : dashed line.

0 to $3 g$ with a fixed frequency $\omega / 2 \pi=30 \mathrm{~Hz}$. Using the parameters defined in Sec. II, the range of the angle parameter was $0.09 \mu^{-1}<\beta<0.7 \mu^{-1}$, and the range of the vibration parameter was $0<\alpha<3.7 \mu^{-1}$. For a friction angle of $40^{\circ}$, the range of accessible parameters is shown as a rectangle in Fig. 2.

The particles are made from crude $\mathrm{Al}_{2} \mathrm{O}_{3}$ alumine powder (BA13 produced by Baikowski), a powder usually used in nuclear industry to simulate the $\mathrm{UO}_{2}$ powder. In a first step, tablets are obtained from uniaxial mechanical compression at $400 \mathrm{MPa}$. These tablets are then sawed in order to obtain two parallel faces. The thickness of the tablet is lower than $500 \mu \mathrm{m}$. This slat is broken to several grains until the size is lower than $500 \mu \mathrm{m}$. Resulting particles hence have two parallel facets and a very angular shape, as can be seen in the picture of Fig. 9. These particles have two preferential facets for sliding, and their large facets prevent an easy tilting of the particle. The horizontal to vertical aspect ratio varies from 5 to 10 from one particle to another. The particle keeps a constant mechanical contact with the substrate. The particles were placed on the

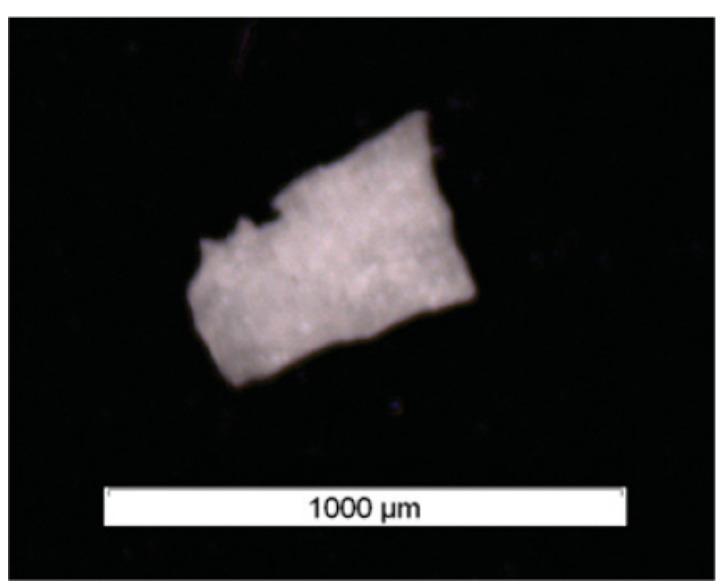

FIG. 9. Photography of a $\mathrm{Al}_{2} \mathrm{O}_{3}$ grain. 
plate with the help of a copper brush to evade electrostatic effects. A great care is taken during experiments to prevent any effect that can alter the friction force between the alumine particle and the substrate, such as the presence of very fine particles emanating from poorly compacted grains.

The motion of the particles down the plane is captured with a Photron high-speed camera with a frame rate of 1000 frames/s and a spatial resolution sufficient to distinguish the rotating grains from the slipping grains. With an appropriate lightening, the particles appear as white objects on a dark background and their positions are easily tracked with the ImageJ image analysis software. The position of the block is compared at each frame with the position of a reference white spot on the plane. The rough trajectory data are altered by roughness variations of the plane surface and a random large-scale motion can be observed. The trajectory is corrected in the $y$ direction by subtracting the floating mean $y$ position (the mean $y$ is computed over a wavelength). This experimental setup allows us to measure the static friction coefficient, the onset of motion predicted by Eq. (6), and to record the block trajectory for various inclination and vibration conditions.

\section{EXPERIMENTAL RESULTS}

\section{A. Static friction coefficient and onset of motion}

As already noticed by various authors, the static friction coefficient $\mu$ is influenced by many parameters (see Table 2 in Blau's paper [1]). Despite this difficulty, we tried to estimate the coefficient of friction between the alumine particle and the mirror-polished stainless-steel plate using two methods. In the first method, the alumine particle is slowly laid on the plate with a fixed angle with the help of a copper brush. When the brush is drawn back, the particle either slides or rests. This is repeated for various increasing angles. In the second method, the particle is laid on the plane at the horizontal then the plane inclination is slowly increased until the particle starts sliding down.

The dispersion of results is rather large since the static friction coefficient depends on the measurement method, on the particle, and also on the location on the plane where the particle is laid. Despite this lack of accuracy, we find a static friction angle $\theta_{c}=30 \pm 5^{\circ}\left(\mu_{s}=0.58 \pm 0.15\right)$.

Expression (6) has been tested experimentally, despite the difficulty of detecting the onset of particle motion. For each inclination angle $\theta<\theta_{c}$, the vibration intensity is gradually raised until the particle motion is detected. To detect the motion, the particle position is measured for each frame by a MATLAB routine and compared to its initial position. When the particle displacement exceeds $50 \mu \mathrm{m}$, the vibration frequency and amplitude is recorded as the vibration threshold. This was repeated five times for each inclination angle, and the results are presented in Fig. 10. The error bars represent the minimum and maximum values of the experimental vibration parameter $\alpha$ measured for each inclination angle. We find a good qualitative agreement between the experimental results and the theory. Most of experimental values of $\alpha$ are larger than the predicted ones. The agreement is the best for low inclination angle $(\beta<0.6)$ where the input vibration energy dominates. For larger inclinations $(\beta>0.6)$, the gravity force

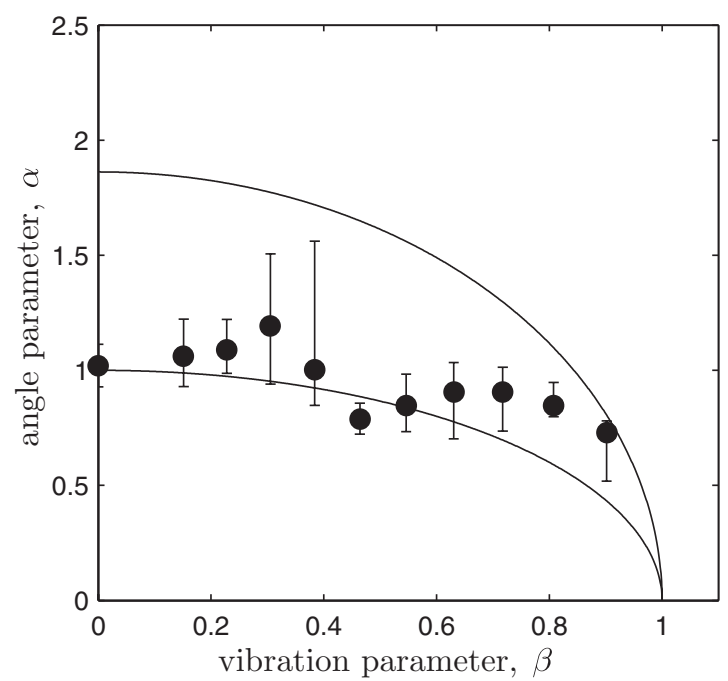

FIG. 10. Comparison of experimental results with expression (6). Solid disks are mean experimental results with five realizations. Angles where $0^{\circ}, 5^{\circ}, 7.5^{\circ}, 10^{\circ}, 12.5^{\circ}, 15^{\circ}, 17.5^{\circ}, 20^{\circ}, 22.5^{\circ}, 25^{\circ}$, and $27.5^{\circ}$.

dominates and the onset of motion seems to be more sensitive to the initial condition and position of the particle.

\section{B. Experimental particle trajectories and dynamic friction coefficient}

An example of a block corrected trajectory is plotted in Fig. 11 (dots connected with a solid line). In this example, the angle parameter is $\beta=0.65$ and the vibration parameter is

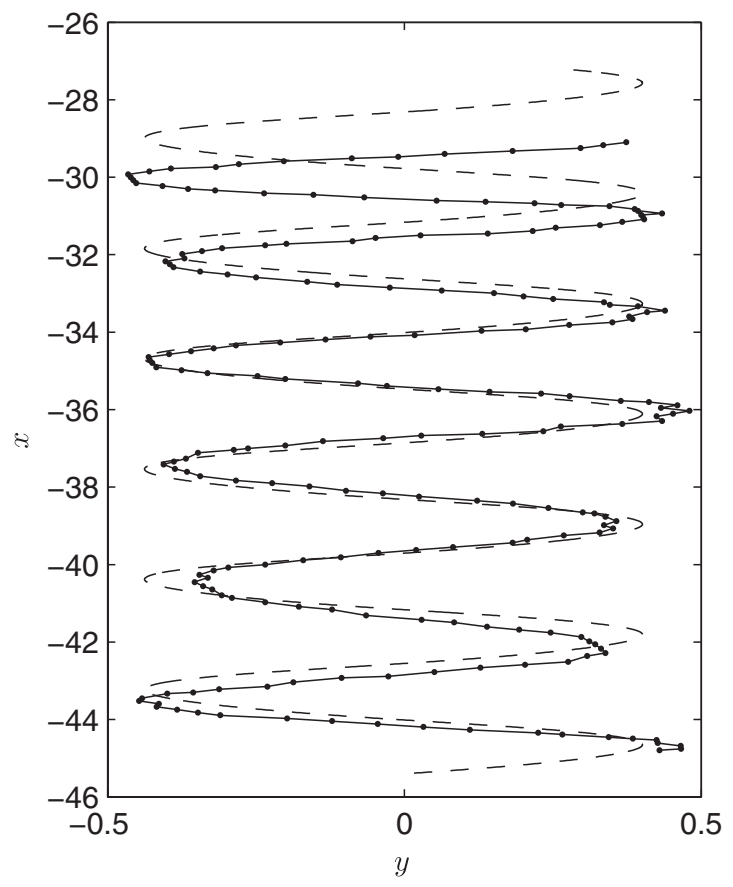

FIG. 11. Comparison between an experimental trajectory (dots linked by a solid line) captured at 1000 frames/s and the corresponding computed trajectory (dashed line) for $\alpha=2.41$ and $\beta=0.65$. Both trajectories are plotted in the laboratory reference frame and correspond to a permanent slip regime. 
$\alpha=2.41$, computed with a friction coefficient $\mu=$ $\tan \left(22.5^{\circ}\right)=0.41$. The corresponding numerical trajectory is plotted as a dashed line in the same figure. There is a very good agreement between the two trajectories, only the phase $\varphi$ of the numerical trajectory has been tuned. The grain amplitude is strikingly similar in the two cases $\left(A_{b}=0.42\right.$ in the simulation and $A_{b}=0.43$ in the experiment), and the wavelengths are also very close with $\lambda=\tau \bar{v}_{x}=2.83$ in the simulation and $\lambda=2.82$ in the experiment. In this example the block is sliding continuously, as predicted by the square symbol in the $(\beta, \alpha)$ map of Fig. 2. As expected, the static coefficient of friction is larger than the dynamic coefficient of friction suitable for a comparison with the numerical model. The dynamic friction coefficient is here an adjustable parameter to find the best fit between the numerical trajectory and the experimental result.

When the vibration intensity is weaker, another behavior can be observed. Figure 12 shows an example of a grain trajectory in the stick-slip regime. The agreement is not as good as in the previous example still a good agreement is found on the trajectory amplitude. The experimental trajectory is rather disymmetric but we have evidence of the stick-slip regime, where the grain is periodically stuck on the plane. These results are obtained for $\alpha=1.00$ and $\beta=0.72$, and the stick-slip regime is again confirmed by the empty circle in the $(\beta, \alpha)$ map of Fig. 2.

The difference between experimental and computed trajectories can be explained by the fact that the particle-to-substrate interaction seems to change during the sliding motion. Despite the care taken during the experiments, the local roughness is not identical all over the plate. In this case, the grain has to

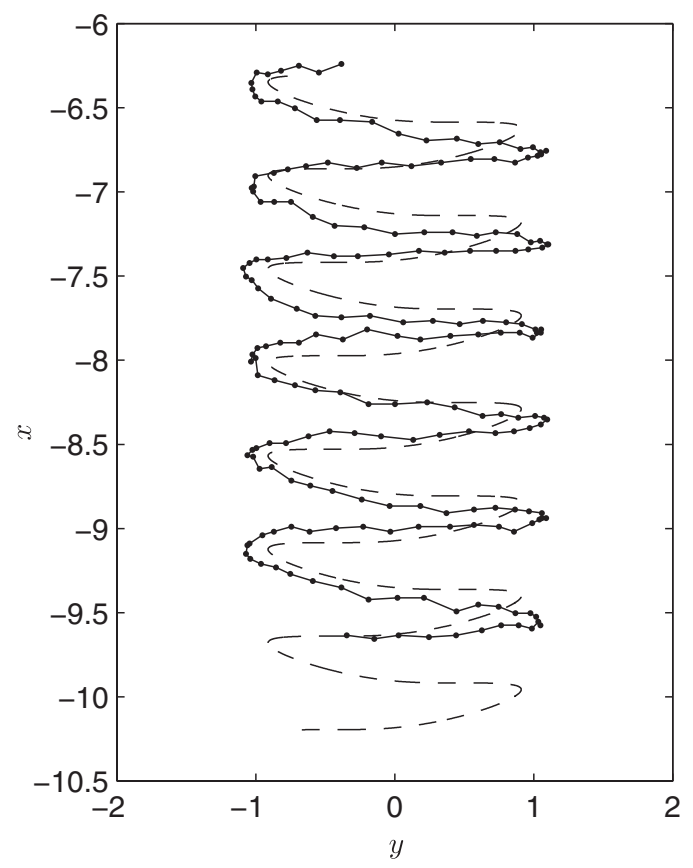

FIG. 12. Comparison between and experimental trajectory (dots linked by a continuous line) captured at 1000 frames/s and the computed trajectory (dashed line) for $\alpha=1.00$ and $\beta=0.72$. Both trajectories are plotted in the laboratory reference frame and correspond to a stick-slip regime.
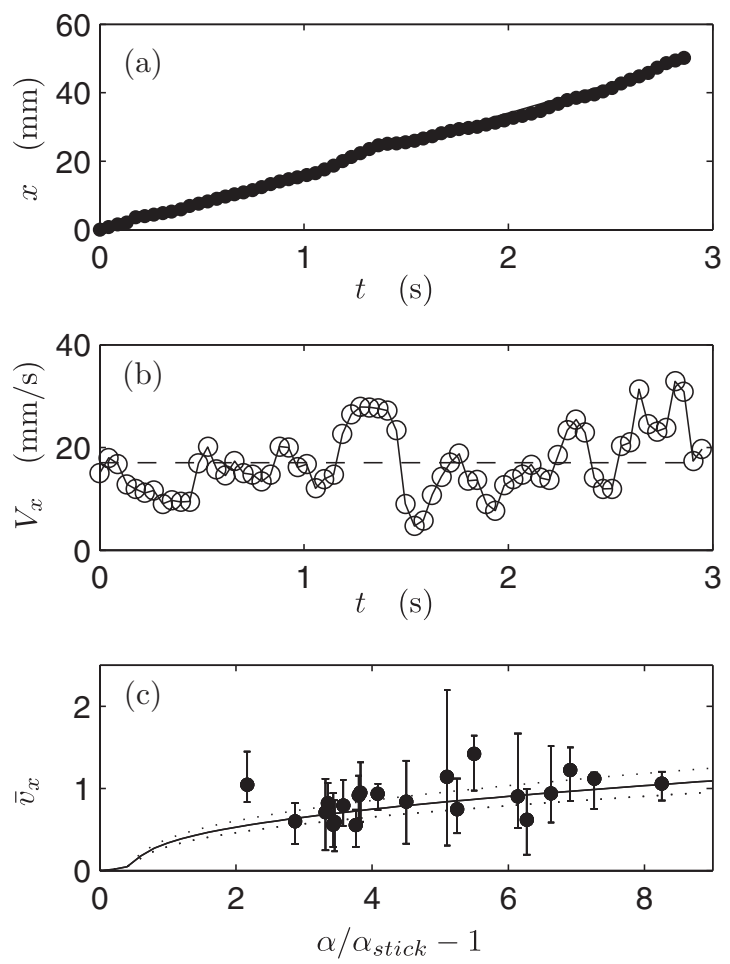

FIG. 13. Example of the downward position of a grain (a) and the corresponding downward velocity (b) during a permanent slip regime for $\alpha=2.6$ and $\beta=0.74$. The velocity is time-averaged over two periods of vibration. The velocity fluctuates around a well defined mean value. Plot (c) shows a comparison between numerical results and experimental results for the mean downward velocity. The continuous line was computed with $\beta=0.7$ and the dotted lines represent the experimental error bar on the angle parameter $\beta=0.7 \pm 0.05$.

overcome the static friction each time it is stuck on the plane. As observed in the previous experiments (Sec. V A), the static friction is very sensitive to the local roughness. This explains the irregularity of the experimental trajectory in Fig. 12.

Figures 13(a) and 13(b) present the mean downward velocity $\bar{v}_{x}$ in the permanent slip regime $(\alpha=2.6$ and $\beta=0.74$ ). This velocity is fluctuating around a mean value [see Fig. 13(b)] but the mean global downward sliding motion is still observed as a periodic regime without mean acceleration. Our experimental results confirm the validity of the single friction coefficient model. From different trajectory measurements, the average dynamic friction coefficient is $\tan \left(20 \pm 5^{\circ}\right)$, lower than the measured static friction coefficient.

The mean downward velocity has been measured experimentally for an inclination parameter $\beta=0.7 \pm 0.05$ and the results are compared with the numerical predictions for a large range of vibration parameter $\alpha$ in Fig. 13(c). On this plot, the continuous line is the velocity $\bar{v}_{x}$ computed for $\beta=0.7$ whereas the lower and upper dotted lines are computed with $\beta=0.65$ and $\beta=0.75$, respectively. The experimental results appear as solid circles with error bars and this plot shows a good agreement with the predicted mean downward velocity. 


\section{CONCLUSION}

The handling and the flow of very angular particles is limited by strong friction effects which can be overcome by the help of transverse vibration. In our particular setup, the slip and stick-slip regimes have been identified and we propose two analytical limiting curves $\alpha_{\text {stick }}(\beta)$ and $\alpha_{\text {slip }}(\beta)$. The validity of the simple theoretical model presented in this paper is assessed by various experimental results. The role of the vibration intensity is negligible when the inclination angle is larger than the friction angle of the particles $(\beta>1)$ but may be very important when the inclination angle is weak. The study of the different energy contributions highlighted a critical value $\beta^{*}=0.722$, below which the transverse vibration plays a major role.
The mean downward velocity $\bar{v}_{x}$ is seen to depend both on the angle parameter $\beta$ and the vibration parameter $\alpha$. A constant mean velocity suggests that the gravity force is balanced by a self-tuned friction force. From a practical point of view, the transport of such an angular particle down a plane is efficient only for $\alpha / \alpha_{\text {stick }} \gtrsim 1.5$. This result is a first step to the understanding of the flow and mass flux of an assembly of angular particles.

Through the comparison of the experimental and numerical trajectories, our vibrated inclined plane setup can be seen to have an indirect measurement device for the dynamic friction coefficient, combining the gravitational and oscillating methods. Despite a lack of accuracy on determining the friction coefficients, this study allows further work on the vibration-induced flow of angular or cohesive powders.
[1] P. J. Blau, Tribol. Int. 34, 585 (2001).

[2] K. Marsui, M. Iura, T. Sasaki, and I. Kokasa, Earthquake Eng. Struct. Dyn. 20, 683 (1991).

[3] B. Westermo and F. Udwadia, Earthquake Eng. Struct. Dyn. 11, 135 (1983).

[4] N. M. Newmark, Geotechnique 15, 139 (1965).

[5] S. L. Kramer and M. W. Smith, J. Geotech. Geoenv. Eng. 123, 635 (2007).

[6] J. J. Thomsen, J. Sound Vib. 228, 1079 (1999).
[7] A. Buguin, F. Brochard, and P.-G. de Gennes, Eur. Phys. J. E 19, 31 (2006).

[8] D. Fleishman, Y. Asscher, and M. Urbakh, J. Phys.: Condens. Matter 19, 096004 (2007).

[9] I. Sánchez, F. Raynaud, J. Lanuza, B. Andreotti, E. Clément, and I. S. Aranson, Phys. Rev. E 76, 060301(R) (2007).

[10] B. C. Méndez, E. Botero, and M. P. Romo, Soil Dyn. Earthquake Eng. 29, 874 (2009). 